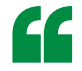

\section{Allocation of astrocytes to restricted regions depending on their point of origin seems to be a general phenomenon throughout the} CNS.
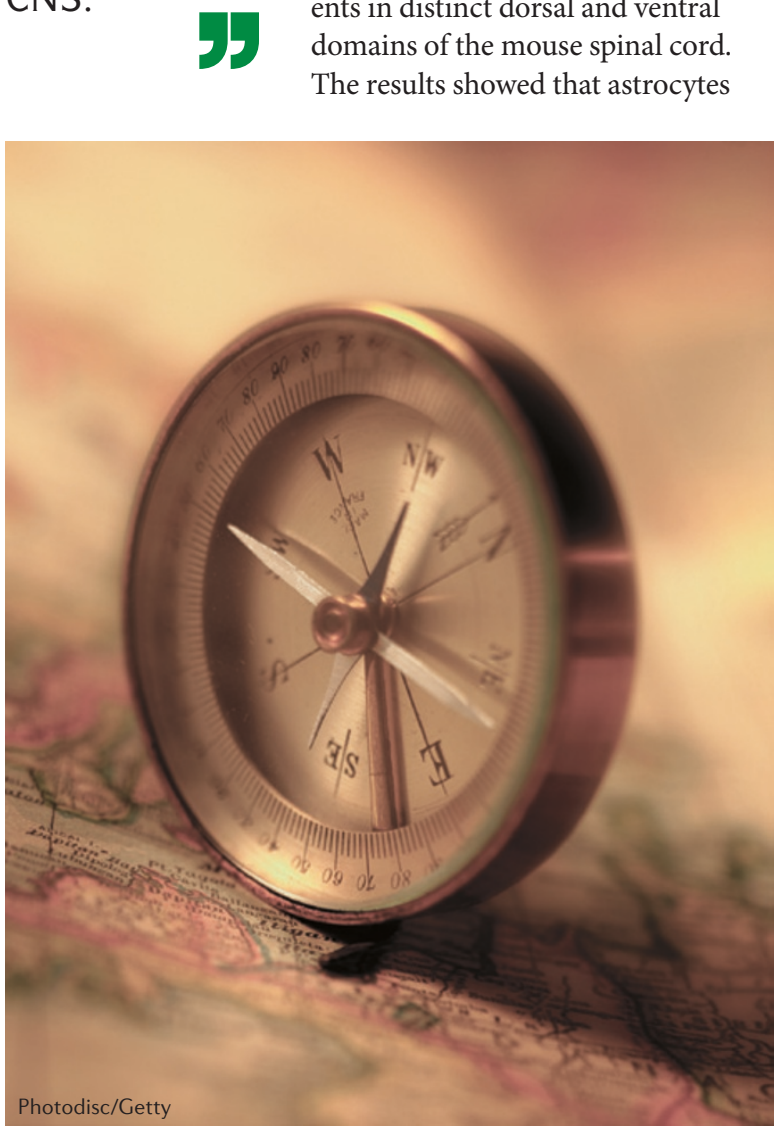

The brain shows regional variations in the electrical properties and gene expression profiles of astrocytes, but it has been unclear how this regional specification arises. New results show that astrocytes in the mouse CNS migrate radially from their points of origin within the ventricular zone to take up positions in restricted spatial domains throughout the brain and spinal cord, with implications for localized astrocyte functions.

Astrocytes arise from radial glia and their progeny. To investigate how astrocytes become restricted to particular regions of the CNS, Tsai et al. used conditional reporter fate mapping of radial glia and their descendents in distinct dorsal and ventral domains of the mouse spinal cord. The results showed that astrocytes

GLIA

\title{
Astrocytes know their place
}

arising from a particular domain of the ventricular zone became localized to a specific region in the spinal cord; for example, astrocytes from the ventral-most ' $\mathrm{p} 3$ ' progenitor domain were always found close to the ventral midline. The radial distribution of astrocytes in the spinal cord depended on the dorsoventral position of their precursors.

To test whether this distribution could be altered, the authors looked at mouse embryos lacking oligodendrocyte transcription factor 2 (Olig2 null embryos), in which the pMN (neuroepithelial progenitor) domain (which usually produces large numbers of oligodendrocyte precursors and few astrocytes) is transformed into a p2-like domain that instead produces astrocytes. Although the oligodendrocyte precursors that are usually produced by the pMN domain migrate widely in all directions, the astrocytes that were generated by this region in Olig2 null embryos remained restricted to a radial distribution, suggesting that this radial distribution is an intrinsic property of astrocytes.

When the authors looked at labelled astrocytes in older mice, they found that the strict regional segregation was maintained; there was no evidence for tangential migration of astrocytes during later stages of development or in adult mice. Even acute injury of the dorsal spinal cord and subsequent gliosis did not induce astrocytes from the neighbouring ventral cord to relocate into the injured dorsal domain. To investigate whether astrocytes fail to migrate into neighbouring domains because those domains already contain a full complement of astrocytes, the authors generated transgenic mice in which astrocytes in the dorsal spinal cord were markedly reduced. Astrocytes from the ventral cord did not migrate into the astrocyte-depleted area. When astrocytes were depleted from around motor neurons, these neurons had fewer excitatory inputs from proprioceptive axons and more inhibitory inputs than expected. This indicates that astrocytes from the correct domain of the ventricular zone are needed to support appropriate synaptogenesis and/or synaptic maintenance in local neuronal populations of the spinal cord.

Labelling of forebrain precursor cells in dorsal, intermediate or ventral ventricular zones showed that astrocytes in the forebrain also migrated radially to take up a dorsoventrally restricted distribution. The authors then used adenovirus-cre infection of foci of radial glia in 1-day-old mice to investigate more-restricted spatial domains and found that labelled astrocytes always followed the trajectories of the processes of the radial glia from which they originated.

These results shed new light on the development of spatially restricted populations of astrocytes and also on their importance for the development and function of the brain and spinal cord. Allocation of astrocytes to restricted regions depending on their point of origin seems to be a general phenomenon throughout the CNS. Without an appropriate population of astrocytes, the profile of synapses on motor neurons is altered, and astrocytes from neighbouring domains are apparently unable to migrate into a depleted or injured area.

Rachel Jones

ORIGINAL RESEARCH PAPER Tsai, H.-H. et al Regional astrocyte allocation regulates CNS synaptogenesis and repair. Science 28 Jun 2012 (doi:10.1126/science.1222381) 